\title{
Development of a local electromagnetic shielding for an extremity magnetic resonance imaging system
}

\author{
Shinya Handa, ${ }^{1, a)}$ Tomoyuki Haishi, ${ }^{2}$ and Katsumi Kose ${ }^{1}$ \\ ${ }_{1}^{1}$ Institute of Applied Physics, University of Tsukuba, Tsukuba, Ibaraki 305-8573, Japan \\ ${ }^{2}$ MRTechnology Inc., Tsukuba, Ibaraki 305-8573, Japan
}

(Received 9 September 2008; accepted 27 October 2008; published online 24 November 2008)

\begin{abstract}
A local radio frequency (rf) shielding consisting of a $\mathrm{Cu}$ plate and an $L C$ balun circuit has been developed for a compact magnetic resonance imaging (MRI) system with a $0.3 \mathrm{~T}$ permanent magnet. Performance of the local $\mathrm{rf}$ shielding was evaluated using an artificial external noise source irradiating a human subject whose hand was inserted into the rf coil of the MRI system. Power spectra of the rf signal detected through the rf coil demonstrated that the local rf shield achieved $30.1 \mathrm{~dB}$ external noise suppression. With the local rf shielding, a MRI of the subject's hand was performed using a three-dimensional gradient-echo sequence. Anatomical structures of the subject's hand were clearly visualized. It was concluded that the local rf shielding could be used for the compact MRI system instead of a rf shielded room. (C) 2008 American Institute of Physics.
\end{abstract}

[DOI: 10.1063/1.3030856]

\section{INTRODUCTION}

Whole body magnetic resonance imaging (MRI) systems are now widely used for clinical diagnosis of various diseases. In addition, dedicated extremity MRI systems have been developed for compactness, low cost, and patient comfort. ${ }^{1-3}$ We have also reported several compact MRI systems dedicated for human extremities. ${ }^{4-7}$

In whole body MRI systems, a radio frequency (rf) shielded room is used to avoid contamination by external noise and to suppress undesired emission from the transmitter coil. Because the large installation space required by a rf shielded room reduces the advantages of the extremity MRI systems, an alternative rf shielding method is highly desired for such MRI systems.

For this reason, commercially available dedicated extremity MRI systems have special designs that do not use rf shielded rooms. The $0.2 \mathrm{~T}$ portable MRI system manufactured by MagneVu has a local surface coil built into the system and does not use a rf shielded room. ${ }^{8,9}$ The $0.18 \mathrm{~T}$ extremity MRI system manufactured by Esaote has rf shielding supplied by a conductive fabric placed around the magnet aperture. ${ }^{10}$ However, the detailed structure and the performance of the rf shielding in those systems have not been reported.

Here we report a design and construction technique for a local rf shielding consisting of a $\mathrm{Cu}$ plate and an $L C$ balun circuit. Utilizing an artificial external noise source, we have quantitatively evaluated the performance of the local rf shielding and demonstrated the usefulness for a compact MRI system with a $0.3 \mathrm{~T}$ permanent magnet.

\footnotetext{
a) Author to whom correspondence should be addressed. Electronic mail: handa@mrlab.frsc.tsukuba.ac.jp.
}

\section{MRI SYSTEM AND LOCAL rf SHIELDING}

\section{A. Compact MRI system}

A compact MRI system with a $0.3 \mathrm{~T}$ permanent magnet was used in this work (Fig. 1). This system was developed for whole hand imaging in our laboratory. ${ }^{11}$ The system consisted of the permanent magnet, gradient coil set, a rf probe, and a compact MRI console. The specifications of the permanent magnet are field strength $=0.3 \mathrm{~T}$, gap width $=140 \mathrm{~mm}$, homogeneity $=23.5 \mathrm{ppm}$ over $20 \times 20 \times 8 \mathrm{~cm}^{3}$ diameter ellipsoidal volume, and total weight $=700 \mathrm{~kg}$.

The rf power transmission and signal receiving system was developed using a DTRX5a MRI transceiver (MRTechnology, Tsukuba, Japan), a narrow bandwidth preamplifier (noise figure $=1.0 \mathrm{~dB}$, gain $=50 \mathrm{~dB} ;$ DST Inc., Asaka, Japan), an active duplexer, and a class $A B$ narrow-band rf

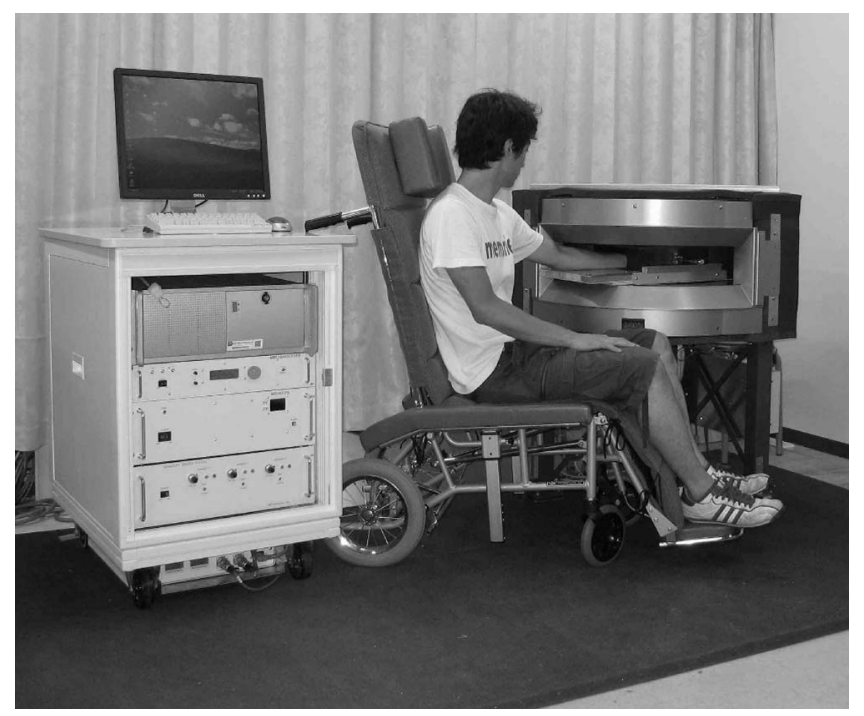

FIG. 1. Overview of the compact MRI system developed and installed in our laboratory. 


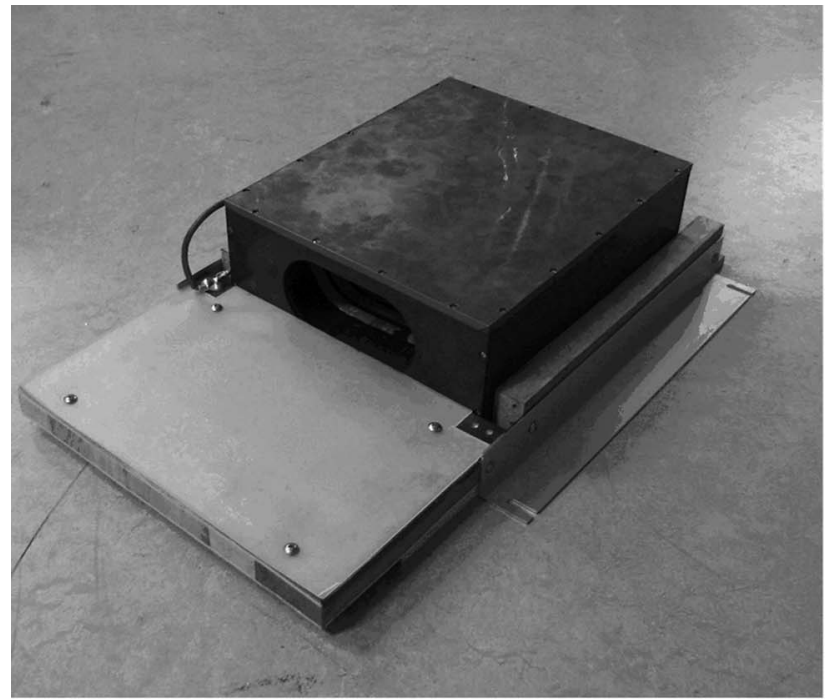

A

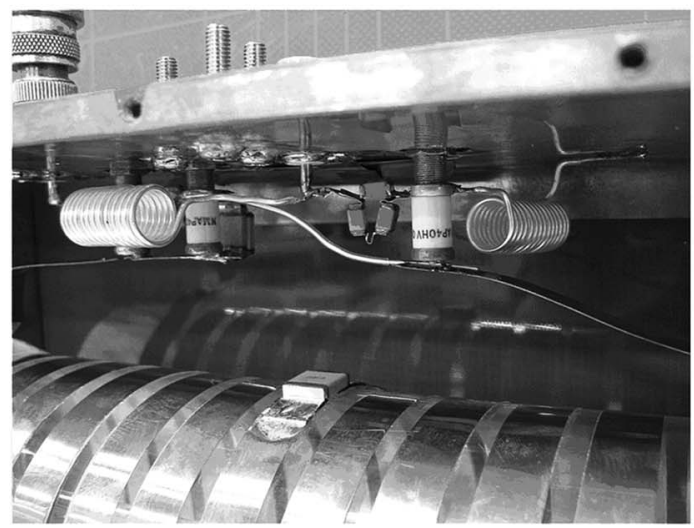

C

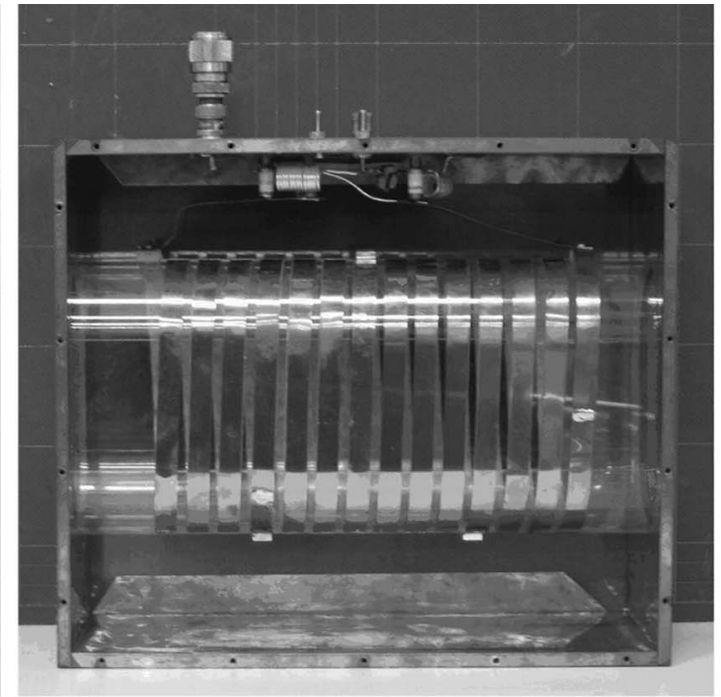

B

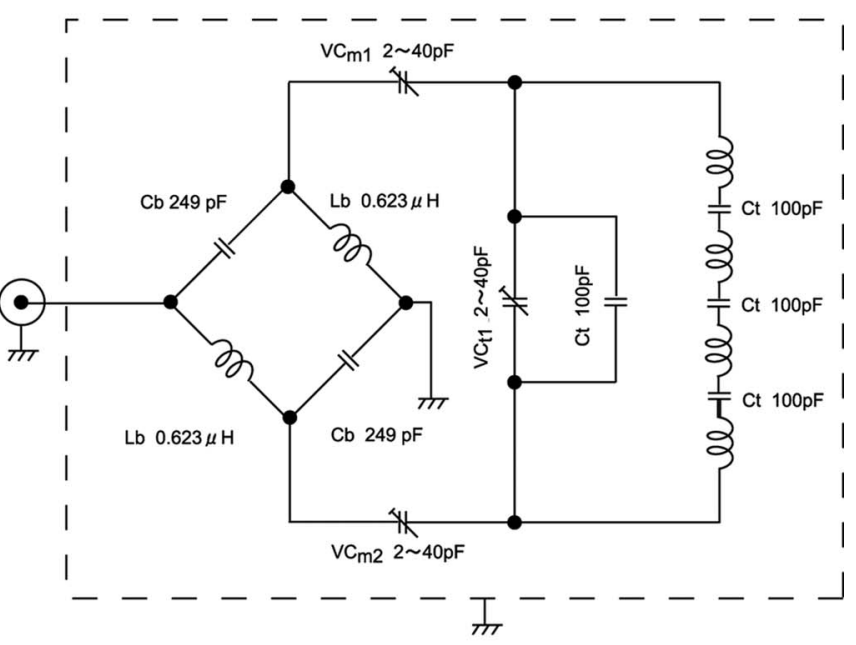

FIG. 2. A rf probe with a local rf shielding developed for hand MR imaging. (a) Overview of the rf probe with an arm-holding table. A Cu plate was built into the table. (b) Inner view of the rf probe box. (c) $L C$ balun attached inside the rf probe box. (d) The circuit of the rf probe.

power amplifier (12.57 MHz, $200 \mathrm{~W}$; DST Inc., Asaka, Japan). The active duplexer consisted of a crossed diodes pair, a quarter-wave line, and a positive-intrinsic-negative diode based rf switcher. The quarter-wave line was made of a double-shielded coaxial cable (3D-2W; characteristic impedance $=50 \Omega$, inner core $=7 \times 0.32 \mathrm{~mm}^{2}$ diameter $\mathrm{Cu}$, outer diameter of dielectric material $=3.0 \mathrm{~mm}$, overall diameter $=6.4 \mathrm{~mm}$ ) to avoid undesired $\mathrm{rf}$ interferences. The active duplexer could be switched on and off in several microseconds.

The rf coil was a 14-turn solenoid optimized for whole hand imaging, with an oval aperture $(7 \mathrm{~cm}$ width $\times 13 \mathrm{~cm}$ height) and $22 \mathrm{~cm}$ length. The coil was wound with a $\mathrm{Cu}$ tape (width $=10 \mathrm{~mm}$, thickness $=0.1 \mathrm{~mm}$ ). The $\mathrm{rf}$ coil was split with three chip capacitors $(100 \mathrm{pF})$ to obtain sharp resonance at $12.58 \mathrm{MHz}$ and to reduce stray capacitance between the rf coil and the hand. Unloaded and loaded $Q$ factors of the rf coil at $12.58 \mathrm{MHz}$ were 260 and 160 . The rf coil was used for both transmission and reception. For electromagnetic stability, the $\mathrm{rf}$ coil was stored in a $\mathrm{rf}$ shielded box made of $100-\mu$ m-thick $\mathrm{Cu}$ sheets attached to 5 -mm-thick bakelite plates.

\section{B. Local rf shielding}

The local rf shielding consisted of a 1-mm-thick $\mathrm{Cu}$ plate and the $L C$ balun circuit. The $\mathrm{Cu}$ plate was placed on an arm-holding table made using a 20-mm-thick wooden block. On the $\mathrm{Cu}$ plate, a 0.5 -mm-thick fiber-reinforced plastic plate was attached to insulate the forearm from the $\mathrm{Cu}$ plate. The $\mathrm{Cu}$ plate was connected to the rf shielded box of the probe. The subject's forearm was connected to the ground by a capacitive coupling to the $\mathrm{Cu}$ plate.

The $L C$ balun circuit was formed as bridge circuit that consisted of inductors $\left(L_{b}\right)$ and capacitors $\left(C_{b}\right)$ in opposing branches. The component values were $L_{b}=0.623 \mu \mathrm{H}$ and $C_{b}=249 \mathrm{pF}$ giving at unity impedance ratio at $12.58 \mathrm{MHz}$. The inductors were 12-turn solenoid coils (diameter 


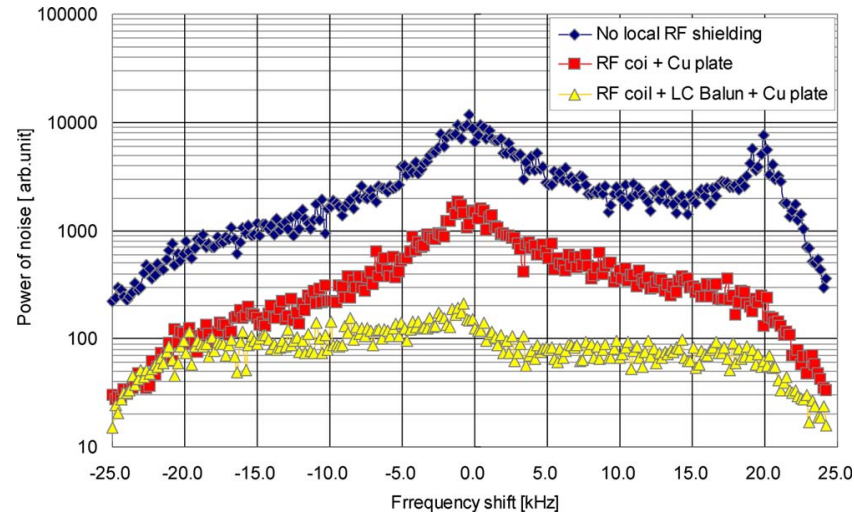

FIG. 3. (Color online) Power spectra of the rf signal detected through the rf coil when an artificial external noise irradiated a human subject. The central frequency is $12.5789 \mathrm{MHz}$.

$=10 \mathrm{~mm}$, length=20 mm) wound with Teflon-coated copper wire (1.0 mm diameter). The $L C$ balun circuit was connected to the rf coil circuit to suppress undesired common mode noise and to balance the rf coil circuit. The entire $L C$ circuit for the rf probe is shown in Fig. 2(b).

\section{EXPERIMENTS}

External noise suppression by the local rf shielding was evaluated using an artificial external noise source and a homebuilt loop antenna. The artificial noise was frequencymodulated Gaussian noise at $12.57950 \mathrm{MHz}$ with $0 \mathrm{dBm}$ intensity generated by a MG3641A signal synthesizer (ANRITSU Inc., Atsugi, Japan). Modulation bandwidth of the artificial noise was $\pm 10 \mathrm{kHz}$.

The loop antenna was made of a three-turn circular loop of coaxial cable $(5 \mathrm{D}-2 \mathrm{~V}$; characteristic impedance $=50 \Omega$, inner core $=1.4 \mathrm{~mm}$ diameter $\mathrm{Cu}$, outer diameter of dielectric material $=4.8 \mathrm{~mm}$, and overall diameter $=7.5 \mathrm{~mm}$ ) and an aluminum box. The diameter of the loop was $15 \mathrm{~cm}$.

A 22-year-old male subject sat on a chair in front of the magnet and inserted his hand into the rf coil. The artificial noise irradiated the subject from the loop antenna located 2.0 $\mathrm{m}$ from the magnet center. Power spectra of the rf signal detected through the rf coil were obtained successively without local rf shielding, with the $\mathrm{Cu}$ plate, and with both the $\mathrm{Cu}$ plate and the $L C$ balun circuit. The power spectra were obtained from Fourier transforms of 2048 digitized points sampled with a dwell time of $20 \mu \mathrm{s}$. Three-dimensional (3D) gradient-echo images $(\mathrm{TR} / \mathrm{TE}=40 / 5 \mathrm{~ms}$, flip angle $=60^{\circ}$, matrix size $=512 \times 256 \times 32$, voxel size $=0.4 \times 0.8$ $\times 1.6 \mathrm{~mm}^{3}$, total acquisition time $=5 \min 30 \mathrm{~s}$ ) of the subject's hand were obtained under the conditions used for the power spectra.

\section{RESULTS AND DISCUSSIONS}

Figure 3 shows the power spectra of the received signal measured under the conditions described in Sec. III. Although the shape of the power spectra was affected by the rf probe frequency response, this figure clearly demonstrates $17.1 \mathrm{~dB}$ noise suppression by the $\mathrm{Cu}$ plate and $30.1 \mathrm{~dB}$ noise suppression by the $\mathrm{Cu}$ plate and the $L C$ balun circuit.
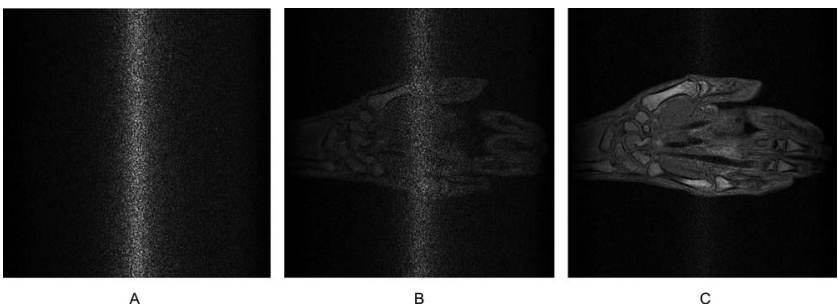

FIG. 4. Coronal sections selected from 3D image data sets of a left hand acquired (a) without the local rf shielding, (b) with the $\mathrm{Cu}$ plate, (c) with both the $\mathrm{Cu}$ plate and the $L C$ balun.

Figure 4 shows two-dimensional coronal cross sections obtained from $3 \mathrm{D}$ data sets acquired with the gradient-echo sequence. These images were selected from the $512 \times 512$ $\times 64$ voxel images reconstructed from the zero-filled Fourier transform of the original $512 \times 256 \times 32$ point signal data set.

Figure 4(a) shows the image acquired without local rf shielding. In this figure, the artificial external noise power was much larger than the MR signal obtained from the subject's hand. Hence, the image of the subject's hand was masked by the noise. Figure 4(b) shows the image acquired with the $\mathrm{Cu}$ plate. Figure 4(c) shows the image acquired with both the $\mathrm{Cu}$ plate and the $L C$ balun circuit. Because the artificial external noise was drastically suppressed, anatomical structures of the subject's hand were clearly visualized. However the artificial noise is still observable. Because the environmental noise power in our laboratory is much smaller than the artificial noise power, it can usually be neglected in imaging experiments in our laboratory environment. These results demonstrate that whole hand imaging is possible using the local rf shielding instead of a rf shielded room.

\section{CONCLUSIONS}

We have developed a local rf shielding using a $\mathrm{Cu}$ plate and an $L C$ balun circuit for a compact MRI system with a 0.3 $\mathrm{T}$ permanent magnet. Performance of the local rf shielding was quantitatively evaluated using an artificial external noise source. The results have demonstrated that the local rf shielding is useful for the elimination of external noise in the compact MRI system.

${ }^{1}$ S. Ghazinoor, J. V. Crues III, and C. Crowley, J. Magn. Reson Imaging 25, 234 (2007).

${ }^{2}$ S. Cohen, Arthritis Rheum. 54, 1034 (2006).

${ }^{3}$ C. Peterfy, T. Roberts, and H. Genant, Radiol. Clin. North Am. 35, 1 (1997).

${ }^{4}$ K. Kose, Y. Matsuda, T. Kurimoto, S. Hashimoto, Y. Yamazaki, T. Haishi, S. Utsuzawa, H. Yoshioka, S. Okada, M. Aoki, and T. Tsuzaki, Magn. Reson. Med. 52, 440 (2004).

${ }^{5}$ N. Iita, S. Handa, S. Tomiha, and K. Kose, Magn. Reson. Med. 57, 272 (2007)

${ }^{6}$ S. Handa, H. Yoshioka, S. Tomiha, T. Haishi, and K. Kose, Magn. Reson. Med. Sci. 6, 113 (2007).

${ }^{7}$ S. Handa, S. Tomiha, K. Kose, and T. Haishi, Magn. Reson. Med. 58, 225 (2007).

${ }^{8}$ G. Gold, D. Theodorou, T. Blair, G. Garcia, C. Crowley, F. Rose, D. Thrudell, and D. Resnick, Proc. Intl. Soc. Mag. Reson. Med. 7, 2039 (1999).

${ }^{9}$ C. W. Crowley and F. H. Rose, U.S. Patent No. 5,304,930 (1994).

${ }^{10}$ A. Carrozzi and L. Spazia, U.S. Patent No. 5,986,531 (1997).

${ }^{11}$ S. Handa, K. Kose, and T. Haishi, Proc. Intl. Soc. Mag. Reson. Med. 16, 1177 (2008) 